\title{
Generalized Dirac Equation with Vector Structural Coefficient and Its Generalized Solutions in Biquaternions Algebra
}

\author{
L.A. Alexeyeva \\ Institute of mathematics and mathematical modeling, Almaty, Kazakhstan.
}

Received: March 17, 2015 / Accepted: April 20, 2015 / Published: August 25, 2015.

\begin{abstract}
On the base of differential biquaternions algebra and theories of generalized functions the biquaternionic wave equation of general type is considered under vector representation of its structural coefficient. Its generalized decisions in the space of tempered generalized functions are constructed. The elementary twistors and twistor fields are built and their properties are investigated. Introduction. The proposed by V.P. Hamilton quaternions algebra [1] and its complex extension - biquaternions algebra are very convenient mathematical tool for the description of many physical processes. At presence these algebras have been actively used in in the work of various authors to solve a number of problems in electrodynamics, quantum mechanics, solid mechanics and field theory. The properties of these algebras are actively studied in the framework of the theory of Clifford algebras. In the papers [2, 3] the differential algebra of biquaternions has been elaborated for construction of generalized solutions of the biquaternionic wave (biwave) equations. The particular types of biwave equations were considered, which are equivalent to the systems of Maxwell and Dirac equations and their generalizations, their biquaternionic decisions also were constructed. Here the biwave equation is considered with vector structural coefficient which is biquaternionic generalization of Dirac equations. Their generalized solutions in the space of tempered distributions are defined and their properties are researched.
\end{abstract}

Keywords: Biquaternionic wave equation, Dirac equation, generalized solution, elementary twistor

\section{Generalized Dirac Equation with Structural Coefficient}

Let's consider the linear biquaternionic differential equations of the type:

$$
\nabla^{ \pm} \mathbf{B}+\mathbf{F} \circ \mathbf{B}=\mathbf{G}(\tau, x),(\tau, x) \in \mathbb{M} .
$$

The structural coefficient $\mathbf{F}=f+F$ is constant biquaternion $(f$ is complex number, $F$ is complex $3 \mathrm{D}$-vector). Biquaternionic multiplications is defined so

$$
\begin{gathered}
\mathbf{F} \circ \mathbf{B}=(f+F) \circ(b+B)= \\
f b-(F, B)+f B+b F+[F, B],
\end{gathered}
$$

where $(F, B),[F, B]$ are the scalar and vector

Corresponding author: L.A. Alexeyeva, Institute of mathematics and mathematical modeling, Almaty, Kazakhstan. E-mail: alexeeva@math.kz. productions of corresponding vectors.

The action of differential biquaternionic operators (mutual bigradients)

$$
\nabla^{ \pm}=\partial_{\tau} \pm i \nabla
$$

are determined as

$$
\begin{gathered}
\nabla^{ \pm} \mathbf{B}(\tau, x) \triangleq\left(\partial_{\tau} \pm i \nabla\right) \circ(b(\tau, x)+B(\tau, x)) \\
\quad=\partial_{\tau} b \mp i \operatorname{div} B \pm i \operatorname{grad} b+\partial_{\tau} B \pm i \operatorname{rot} B
\end{gathered}
$$

(accordingly to upper or lower sign), $\nabla=\left(\partial_{1}, \partial_{2}, \partial_{3}\right)$ is gradient, $\mathbb{M}$ is Minkowski space.

Since the equation (1) is equivalent to systems of hyperbolic type and brings to solving the wave equations, we name them biwave equations of general type. Special cases of this equation when $\mathbf{F}=0 \quad$ и $\quad \mathbf{F}=f \quad$ is complex number give biquaternionic representation of Maxwell and Dirac 
equations.

Here we consider the case when $\mathbf{F}=F$ is complex vector. Hereinafter we 'll build the decisions of the equation (1) for upper sign (+). The decisions for lower sign (-) possible to get of them by use of the operation of complex conjugation.

In this case the Eq. (1) has have the form:

$$
\left(\partial_{\tau}+i \nabla+F\right) \mathbf{B}=\mathbf{G} .
$$

We'll construct the solutions of this equation with use of theory of generalized functions [4].

\section{Generalized Solutions of Dirac Equation with Vector Structural Coefficient}

Let's introduce the mutual operators of the type

$$
\mathbf{D}_{F}^{+}=\nabla^{+}+F, \quad \mathbf{D}_{F}^{-}=\nabla^{-}-F .
$$

Easy to check that their superposition is commutative and possesses following useful property

$$
\begin{aligned}
\mathbf{D}_{F}^{+} \mathbf{D}_{F}^{-}= & \mathbf{D}_{F}^{-} \mathbf{D}_{F}^{+}=\left(\nabla^{+}+F\right) \circ\left(\nabla^{-}-F\right)= \\
& =\square+(F, F)+2 i(F, \nabla),
\end{aligned}
$$

where $\square \triangleq \partial_{\tau}^{2}-\Delta$ is wave D'Alembert's operator, $\Delta$ is Laplace operator. Using (3) we get from (2)

$$
\mathbf{D}_{F}^{-} \mathbf{D}_{F}^{+} \mathbf{B}=\{\square+(F, F)+2 i(F, \nabla)\} \mathbf{B}=\mathbf{D}_{F}^{-} \mathbf{G}=\mathbf{Q} .
$$

I.e. each component $\mathbf{B}$ satisfies to the equation

$$
\square u+(F, F) u+2 i(F, \nabla u)=q(\tau, x)
$$

with corresponding component of $\mathbf{Q}$ in right part.

Theorem 1. Decisions of generalized biwave equation (2) possible to present in the form

$$
\mathbf{B}=\mathbf{D}_{F}^{-} \psi * \mathbf{G}+\mathbf{B}^{0}=\psi * \mathbf{D}_{F}^{-} \mathbf{G}+\mathbf{B}^{0},
$$

where $\psi(\tau, x)$ are fundamental decisions of Eq. (4) (for $q=\delta(\tau) \delta(x))$, and $\mathbf{B}^{0}(\tau, x)$ is solutions of homogeneous Eq. (2) (for $\mathbf{G}=0)$ :

$$
\begin{gathered}
\mathbf{B}^{0}=\sum_{\psi^{0}} \mathbf{D}_{F}^{-} \psi^{0} * \mathbf{C}^{0}=\sum_{\psi^{0}} \psi^{0} * \mathbf{D}_{F}^{-} \mathbf{C}^{0} \\
=\sum_{\psi^{0}} \mathbf{D}_{F}^{-}\left(\psi^{0} * \mathbf{C}^{0}\right)
\end{gathered}
$$

$\psi^{0}(\tau, x)$ are solutions of homogeneous scalar Eq. (4), $C^{0}(\tau, x)$ are arbitrary biquaternions which allow such convolution.

Proof. In virtue of linearity of the Eq. (1), it is enough to prove the statement for each summand in formula (5). We substitute first summand in equation (2) and, using (3), get

$$
\begin{gathered}
\left(\nabla^{+}+F\right)\left(\nabla^{-}-F\right)(\psi * \mathbf{G})= \\
(\square \psi+2 i(F, \nabla \psi)+(F, F) \psi) * \mathbf{G}= \\
\delta(\tau) \delta(x) * \mathbf{G}=\mathbf{G} .
\end{gathered}
$$

For each summand of second sum we have

$$
\begin{gathered}
\left(\nabla^{+}+F\right)\left(\nabla^{-}-F\right)\left(\psi^{0} * \mathbf{C}^{0}\right)= \\
\left\{\square \psi^{0}+2 i\left(F, \nabla \psi^{0}\right)+(F, F) \psi^{0}\right\} * \mathbf{C}^{0}=0 .
\end{gathered}
$$

Here we used the known properties of convolutions [4]. Obviously, in the virtue of of linearity of the equation, any decision possible to present in similar form.

Let's build these solutions.

\section{Scalar Potentials of Inhomogeneous Scalar Equation (4). Twistor}

From theorem 1.1 follow that to determine solutions of Eq.(1) it's necessary to find solutions of scalar inhomogeneous $(q(\tau, x) \neq 0)$ and homogeneous $(q(\tau, x)=0)$ equation (4).

Theorem 2. Fundamental decisions of the equation (4) may be presented in the form

$$
\begin{gathered}
\psi(\tau, x)= \\
\frac{e^{i(F, x)}}{4 \pi\|x\|}((1-a) \delta(\tau-\|x\|)+a \delta(\tau+\|x\|))+\psi^{0}, \\
\forall a \in \mathbb{Z},
\end{gathered}
$$

where $\delta(\tau \mp\|x\|)$ is simple layer on light cone $\tau= \pm\|x\|$; constant $a$ is arbitrary complex number, $\psi^{0}(\tau, x)$ is solution of homogeneous Eq. (4) (for 
$q=0)$.

Proof. For proof of this formula we use the Fourier transform of generalized functions. Hereinafter Fourier variables corresponding to $(\tau, x)$ we mark $(\omega, \xi)$ accordingly.

Equation for $\psi$ has the form

$$
\square \psi+(F, F) \psi+2 i(F, \nabla \psi)=\delta(\tau) \delta(x),
$$

and its Fourier transform is

$$
\left(\|\xi\|^{2}-\omega^{2}+2(F, \xi)+(F, F)\right) \bar{\psi}(\omega, \xi)=1 .
$$

From here we get

$$
\bar{\psi}(\omega, \xi)=\frac{1}{(\xi+F, \xi+F)-\omega^{2}} .
$$

Since it has have nonintegrable singularities, for building of the Fourier reconversion it's need to choose the certain regularization of the right part.

For this we use the fundamental decision of D'Alembert equation

$$
\square \chi=\delta(\tau, x),
$$

which has have the form

$$
\begin{gathered}
\chi=\frac{1-a}{4 \pi\|x\|} \delta(\tau-\|x\|)+\frac{a}{4 \pi\|x\|} \delta(\tau+\|x\|), \\
\forall a \in \mathbb{Z} .
\end{gathered}
$$

Here singular generalized function $\delta(\tau \pm\|x\|)$ is simple layer on the light cone $\tau=\mp\|x\|$.

As Fourier transforms of summands are equal to the such regularizations :

$$
\mathrm{F}\left[\frac{1}{4 \pi\|x\|} \delta(\tau \mp\|x\|)\right]=\frac{1}{\|\xi\|^{2}-\omega^{2} \pm i 0},
$$

using property of a shift of Fourier transform [4], from (10) and (11) we get the formula of the theorem (7). Theorem is proved.

Note that $\psi$ is the spherical wave, spreading in $R^{3}$ with unit velocity (if $\tau$ is time). $\operatorname{Im} F$ gives exponential decreasing or increasing to density depending on directions $x$ to $F \cdot \operatorname{Re} F$ defines density oscillation along it. By this cause we name Eq.(1) as twistors equation and its decisions twistors.

\section{Scalar Potentials of Homogeneous Twistors Equation}

Let's construct the solutions of homogeneous equation (4):

$$
\square \psi^{0}+(F, F) \psi^{0}+2 i\left(F, \nabla \psi^{0}\right)=0
$$

Its Fourier transform has the form:

$$
\left((\xi+F, \xi+F)-\omega^{2}\right) \bar{\psi}^{0}=0 .
$$

Consequently $\quad \bar{\psi}^{0}=\phi(\omega, \xi) \delta_{S}(\omega, \xi) \quad, \quad$ where $\delta_{S}(\omega, \xi)$ is simple layer on $3 \mathrm{D}$-surface $S$ in $R^{4}$ :

$$
S=\left\{(\omega, \xi):(\xi+F, \xi+F)-\omega^{2}=0\right\},
$$

and $\phi(\omega, \xi)$ is arbitrary integrable function on $S$.

Then formal decision of the uniform equation has have the form of surface integral

$$
\begin{gathered}
\psi^{0}(\tau, x)= \\
\int_{S} \phi(\omega, \xi) \exp (-i \omega \tau-i(x, \xi)) d S(\omega, \xi), \\
\forall \phi(\omega, \xi) \in L_{1}(S) .
\end{gathered}
$$

Let's study, under what $F$ does such surface exist and what type does it have?

4.1 Let $F$ is real vector $F=-E$. Then $S$ is a cone in $R^{4}$ with the top in the point $(\omega, \xi)=(0, E)$. In this case the form (14) may be written as

$$
\begin{gathered}
\psi^{0}(\tau, x)= \\
\int_{R^{3}} \chi(\xi) \exp ( \pm i\|\xi-E\| \tau-i(x, \xi)) d V(\xi), \\
\forall \chi(\xi) \in L_{1}\left(R^{3}\right)
\end{gathered}
$$




$$
d V(\xi)=d \xi_{1} d \xi_{2} d \xi_{3}
$$

4.2 If $F$ is imaginary vector $F=-i H$ then from Eq.(13) follow

$$
(\xi-i H, \xi-i H)=\|\xi\|^{2}-\|H\|^{2}-2 i(H, \xi)=\omega^{2} .
$$

The decision of this equation is two sets intersection :

$$
S=\left\{(\omega, \xi):\|\xi\|^{2}-\|H\|^{2}=\omega^{2}, \quad(H, \xi)=0\right\}
$$

In this case the decision of the uniform equation is an integral on a part of the planes, perpendicular to vector $H$, without circle, center of which is in the point $\xi=0$, radius is equal to $\|H\|$ :

$$
\begin{gathered}
\psi^{0}(\tau, x)= \\
\int_{\substack{\xi \perp H,\|\xi\| \geq H \|}} \chi(\xi) \exp \left( \pm i \tau \sqrt{\|\xi\|^{2}-\|H\|^{2}}-i(x, \xi)\right) d S_{\cap}(\xi),
\end{gathered}
$$$$
\forall \chi(\xi) \in L_{1}\left(S_{\curvearrowleft}(\xi)\right)
$$

4.3 If $F=-E-i H$ is complex vector, then from (13) follow:

$$
\begin{gathered}
(\xi-E-i H, \xi-E-i H)= \\
\|\xi-E\|^{2}-\|H\|^{2}-2 i(\xi-E, H)=\omega^{2} .
\end{gathered}
$$

In this case the decision of the homogeneous equation is an integral on a part of the planes, perpendicular to vector $H$, without circle, center of which is in the point $\xi^{*}$, radius is equal to $\|H\|$ :

$$
\begin{gathered}
\psi^{0}(\tau, x)= \\
\int_{\substack{(\xi-E) \perp H,\|\xi-E\| \geq\|H\|}} \varphi(\xi) \exp \left( \pm i \tau \sqrt{\|\xi-E\|^{2}-\|H\|^{2}}-i(x, \xi)\right) d S_{\cap}(\xi), \\
\forall \varphi(\xi) \in L_{1}\left(S_{\cap}(\xi)\right) .
\end{gathered}
$$

Here

$$
S_{\cap}(\xi)=\{\xi:(\xi-E) \perp H \cap\|\xi-E\| \geq\|H\|\} .
$$

The choice $\varphi(\xi)$ allows to build a broad class of the decisions of the twistor equations.

\section{Elementary $\xi$-Twistor. Nonstationary Twistor Fields}

Note that the subintegral function in the formula (17)

$$
\begin{gathered}
\psi_{\xi}^{ \pm}(\tau, x)= \\
\exp \left( \pm i \tau \sqrt{\|\xi-E\|^{2}-\|H\|^{2}}-i(x, \xi)\right), \\
\xi \in S_{\curvearrowleft}(\xi)
\end{gathered}
$$

are also solutions of uniform Eq.(4) by constructing. They present two plane harmonic waves, moving in direction wave vector $\xi$ with phase velocity

$$
c=\sqrt{\|\xi-E\|^{2}-\|H\|^{2}} /\|\xi\|
$$

wave length

$$
\lambda=2 \pi /\|\xi\|
$$

5.1 For $\|\xi-E\|>\|H\|$ their frequency

$$
\varpi=\sqrt{\|\xi-E\|^{2}-\|H\|^{2}},
$$

the period

$$
T=2 \pi / \sqrt{\|\xi-E\|^{2}-\|H\|^{2}} .
$$

Let's calculate generated by them elementary $\xi$-twistors:

$$
\begin{gathered}
\Psi_{\xi}^{ \pm}=\frac{1}{\sqrt{2}\|\xi-E\|} \mathbf{D}_{F}^{-} \psi_{\xi}^{ \pm}= \\
\frac{ \pm i \sqrt{\|\xi-E\|^{2}-\|H\|^{2}}-(\xi-E)+i H}{\sqrt{2}\|\xi-E\|} \psi_{\xi}^{ \pm},
\end{gathered}
$$

$$
\xi \neq E
$$

Its norm and pseudonorm [1,2] are 


$$
\left\|\Psi_{\xi}^{ \pm}\right\|=1,\left\langle\left\langle\Psi_{\xi}^{ \pm}\right\rangle\right\rangle=i \frac{\|H\|}{\|\xi-E\|}
$$

Biquaternion of energy-impulse $[1,2]$

$$
\Xi\left(\Psi_{\xi}^{ \pm}\right)=W\left(\Psi_{\xi}^{ \pm}\right)+i P\left(\Psi_{\xi}^{ \pm}\right)=\Psi_{\xi}^{ \pm} \circ\left(\Psi_{\xi}^{ \pm}\right)^{*}
$$

is equal to

$$
\begin{gathered}
\Xi\left(\Psi_{\xi}^{ \pm}\right)= \\
1+i \frac{\left[e_{\xi-E}, H\right] \pm e_{\xi-E} \sqrt{\|\xi-E\|^{2}-\|H\|^{2}}}{\|\xi-E\|} \\
e_{\xi-E}=\frac{\xi-E}{\|\xi-E\|}
\end{gathered}
$$

The norm and pseudonorm $\Xi_{\xi}$ are equal to

$$
\begin{gathered}
\left\|\Xi\left(\Psi_{\xi}^{ \pm}\right)\right\|=\sqrt{1+\frac{\|\xi-E\|^{2}-\|H\|^{2} \cos ^{2} \gamma}{\|\xi-E\|^{2}}} \\
\left\langle\left\langle\Xi\left(\Psi_{\xi}^{ \pm}\right)\right\rangle\right\rangle=\sqrt{1-\frac{\|\xi-E\|^{2}-\|H\|^{2} \cos ^{2} \gamma}{\|\xi-E\|^{2}}}
\end{gathered}
$$

where $\gamma$ is the angle between vectors $e_{\xi-E}, H$. It's interesting that by $\gamma= \pm \pi / 2$

$$
\left\langle\left\langle\Xi\left(\Psi_{\xi}^{ \pm}\right)\right\rangle=0, \quad\left\|\Xi\left(\Psi_{\xi}^{ \pm}\right)\right\|=\sqrt{2} .\right.
$$

The simplest type $\Psi_{\xi}^{ \pm}$и $\Xi\left(\Psi_{\xi}^{ \pm}\right)$have by $H=0$ :

$$
\begin{gathered}
\Psi_{\xi}^{ \pm}=\frac{ \pm i+e_{\xi-E}}{\sqrt{2}} \exp ( \pm i \tau\|\xi-E\|-i(x, \xi)) \\
\left\|\Psi_{\xi}^{ \pm}\right\|=1, \quad\left\langle\left\langle\Psi_{\xi}^{ \pm}\right\rangle\right)=0, \quad \Xi\left(\Psi_{\xi}^{ \pm}\right)=1 \pm i e_{\xi-E} .
\end{gathered}
$$

Elementary $\xi$-twistors describe the 8D-plane harmonic waves, moving in direction of vector $\xi$. Their amplitudes depend on scalar and vector real and imaginary part of a twistor. The direction of the motion is determined by upper or lower sign of the twistor and influences upon amplitude of the wave.

Twistors fields. Using $\Psi_{\xi}^{ \pm}$it may present $\mathbf{B}^{0}(\tau, x)$ as the sum of twistors:

$$
\mathbf{B}^{\xi}(\tau, \mathbf{x})=\Psi_{\xi}^{+} * \mathbf{C}_{1}(\tau, \mathbf{x})+\Psi_{\xi}^{-} * \mathbf{C}_{2}(\tau, \mathbf{x}),
$$

which describe $\xi$-polarize twistors fields.

Depolarize twistors fields are described of the biquaternions of the type

$$
\begin{gathered}
\mathbf{B}^{0}(\tau, \mathbf{x})=\sum_{\mathbf{C}, \varphi} \Psi^{\varphi}(\tau, \mathbf{x}) * \mathbf{C}(\tau, \mathbf{x}), \\
\Psi^{\varphi}(\tau, x)=\int_{S_{\cap}} \varphi(\xi) \Psi_{\xi}(\tau, x) d S_{\curvearrowleft}(\xi), \\
\forall \varphi \in L_{1}\left(S_{\cap}\right) .
\end{gathered}
$$

Here scalar-vector fields $\mathbf{C}_{j}(\tau, x)$ are arbitrary generalized biquaternions [1], which admit such convolutions.

\section{Standing twistors and twistors fields}

By $\|\xi-E\| \leq\|H\|$ scalar potential (18) is converted to the function:

$$
\begin{gathered}
\alpha_{\xi}^{ \pm}(\tau, x)=\exp \left( \pm \tau \sqrt{\|H\|^{2}-\|\xi-E\|^{2}}-i(x, \xi)\right), \\
(\xi-E) \perp H .
\end{gathered}
$$

It is also the solution of Eq. (12). These are two standing harmonic waves with exponential decreasing and increasing amplitude during the time. By $\|\xi-E\|=\|H\|$ the amplitude does not depend on the time and they coincide.

Let's calculate generated $\alpha_{\xi}^{ \pm}$elementary twistor:

$$
\begin{gathered}
\Upsilon_{\xi}^{ \pm}(\tau, x)=\frac{\mathbf{D}_{F}^{-} \alpha_{\xi}^{ \pm}}{\|\xi-E\| \sqrt{2}}= \\
\frac{ \pm \sqrt{\|H\|^{2}-\|\xi-E\|^{2}}-(\xi-E)+i H}{\|\xi-E\| \sqrt{2}} \alpha_{\xi}^{ \pm}(\tau, x), \\
(\xi-E) \perp H
\end{gathered}
$$

Its norm and pseudonorm are equal to

$$
\left\|\Upsilon_{\xi}^{ \pm}\right\|=\frac{\|H\|}{\|\xi-E\|},\left\langle\left\langle\Upsilon_{\xi}^{ \pm}\right\rangle\right\rangle=i
$$


Its energy-impulse biquaternion is

$\Xi\left(\Psi_{\xi}^{ \pm}\right)=1+\frac{i\left[e_{\xi-E}, H\right] \pm e_{\xi-E} \sqrt{\|H\|^{2}-\|\xi-E\|^{2}}}{\|\xi-E\|}$

Similarly (23) it's possible to build exponentially increasing or decreasing on time twistor fields from polarized ones of type

$$
\mathbf{B}^{\xi}(\tau, \mathbf{x})=\mathbf{\Upsilon}_{\xi}^{ \pm} * \mathbf{C}(\tau, \mathbf{x})
$$

or depolarize field of kind

$$
\begin{aligned}
& \mathbf{B}^{0}(\tau, \mathbf{x})=\sum_{\mathbf{C}, \varphi} \mathbf{\Upsilon}^{\varphi}(\tau, \mathbf{x}) * \mathbf{C}(\tau, \mathbf{x}), \\
& \mathbf{\Upsilon}^{\varphi}(\tau, x)=\int_{S_{\curvearrowleft}} \varphi(\xi) \mathbf{\Upsilon}_{\xi}(\tau, x) d S_{\curvearrowleft}(\xi), \\
& \forall \varphi \in L_{1}(\xi \perp H) .
\end{aligned}
$$

5.2 If $\xi=E$, from scalar potential (18) we get elementary $H$-twistor:

$$
\begin{gathered}
\Psi_{H}^{ \pm}=\frac{1}{\sqrt{2}\|H\|} \mathbf{D}_{F}^{-} \exp (\mp \tau\|H\|-i(E, x))= \\
\frac{\left(\mp 1+i e_{H}\right)}{\sqrt{2}} \exp (\mp \tau\|H\|-i(E, x)), \\
\left\|\Psi_{H}^{ \pm}\right\|=1, \quad\left\langle\left\langle\Psi_{H}^{ \pm}\right\rangle\right\rangle=0, \quad \Xi\left(\Psi_{H}^{ \pm}\right)=\sqrt{2} .
\end{gathered}
$$

\section{Conclusion}

Here it's shown that for twistors there are generating their scalar potentials which satisfy to waves equation. The decisions of this equation gives possibility to construct elementary twistors, and on their base the wide class of twistors fields which possible to use in elementary particles theory.

This theory can be used for construction of solutions of the equations of charge-currents transformation under action of external electro-gravimagnetic fields for a one biquaternionic model of EGM-fields and their interactions, which was elaborated by author earlier [5, $6]$.

\section{References}

[1] Hamilton W.R. On a new Species of Imaginary Quantities connected with a theory of Quaternions, Proceedings of the Royal Irish Academy, 2 (1843), 424-434.

[2] Alexeyeva L.A. Differential algebra of biquaternions// Mathematical journal, 10(2010), No 1, 33-41; 10(2010), No 4,5-13; 11(2011), No 1, 33-41. (See www.math.kz in Russian)

[3] Alexeyeva L.A. Biquaternions algebra and its applications by solving of some theoretical physics equations//Clifford Analysis, Clifford algebras and their applications. 7 (2012), No 1, 19-39.

[4] Vladimirov V.S. Generalized functions in mathematical physics. Moscow: Science, 1976,512.

[5] Alexeyeva L.A. Fields analogue of Newton lows for a one model of electro-gravimagnetic field //Hypercomplex number in geometry and physics. 6(2009), No 1, 122-134 (in Russian).

[6] Alexeyeva L.A. Newton's laws for a biquaternionic model of the electro-gravimagnetic fields, charges, currents, and their interactions //Ashdin publishing. Journal of Physical Mathematics. 1(2009), article ID S090604. 